\title{
Control of Toxin-Antitoxin Systems by Proteases in Mycobacterium Tuberculosis
}

\author{
Patricia Bordes * and Pierre Genevaux* \\ Laboratoire de Microbiologie et de Génétique Moléculaires, Centre de Biologie Intégrative, Université de Toulouse, CNRS, UPS, \\ Toulouse, France
}

Toxin-antitoxin (TA) systems are small genetic elements composed of a noxious toxin and a counteracting cognate antitoxin. Although they are widespread in bacterial chromosomes and in mobile genetic elements, their cellular functions and activation mechanisms remain largely unknown. It has been proposed that toxin activation or expression of the TA operon could rely on the degradation of generally less stable antitoxins by cellular proteases. The resulting active toxin would then target essential cellular processes and inhibit bacterial growth. Although interplay between proteases and TA systems has been observed, evidences for such activation cycle are very limited.

OPEN ACCESS

Edited by: Matthias Peter Mayer, Heidelberg University, Germany

Reviewed by: Christina Bourne, University of Oklahoma, United States Teru Ogura, Kumamoto University, Japan

*Correspondence: Patricia Bordes patricia.bordes@univ-tlse3.fr Pierre Genevaux pierre.genevaux@univ-tlse3.fr

Specialty section: This article was submitted to Protein Folding, Misfolding and Degradation, a section of the journal Frontiers in Molecular Biosciences

Received: 06 April 2021 Accepted: 06 May 2021

Published: 17 May 2021

Citation: Bordes P and Genevaux P (2021) Control of Toxin-Antitoxin Systems by

Proteases in

Mycobacterium Tuberculosis. Front. Mol. Biosci. 8:691399. doi: 10.3389/fmolb.2021.691399 Herein, we present an overview of the current knowledge on TA recognition by proteases with a main focus on the major human pathogen Mycobacterium tuberculosis, which harbours multiple TA systems (over 80), the essential AAA + stress proteases, ClpC1P1P2 and ClpXP1P2, and the Pup-proteasome system.

Keywords: AAA+ proteases, proteasome, toxin-antitoxin system, mycobacterium, protein degradation

\section{GENERAL OVERVIEW OF TOXIN ANTITOXIN SYSTEMS IN $M$. TUBERCULOSIS}

The bacterium Mycobacterium tuberculosis, the causative agent of tuberculosis, is a major public health problem accounting for over 1.5 million deaths per year. The emergence of multidrug resistant (MDR) and extensively drug-resistant (XDR) Mtb strains has significantly challenged current tuberculosis treatments and increase the need for new treatment strategies (WHO report 2018; www.who.int/tb/data). The ability to sense and tolerate multiple host derived stresses, evade host defenses and persist within infected hosts is central to the pathogenicity of $M$. tuberculosis. Therefore, deciphering molecular mechanisms underlying stress tolerance and sensing in M. tuberculosis is critical for developing new strategies to fight tuberculosis.

M. tuberculosis strains possess a remarkably high number of toxin-antitoxin (TA) systems in their genome (Ramage et al., 2009; Akarsu et al., 2019; Tandon et al., 2019). As an example, the most studied laboratory strain H37Rv encodes for more than 80 TA systems and it has been proposed that such systems could contribute to it pathogenesis (Sala et al., 2014). Classical TA systems are small genetic modules composed of a deleterious toxin and an antitoxin that neutralizes the effects of the toxin. TA systems are organized into operons and are widely distributed throughout the bacterial genome (Van Melderen, 2010). Toxins generally target essential functions of the host bacterium, such as translation, replication, membrane integrity or peptidoglycan synthesis, causing growth to slow down and eventually leading to cell death (Page and Peti, 2016; Harms et al., 2018; Wilmaerts et al., 2018). TA systems are often found on plasmids, for which they were designated as addiction 
modules since they are involved in their stabilization by inhibiting growth of daughter cells that would not have inherited the plasmid carrying the TA system (Ogura and Hiraga, 1983). The roles of chromosomal TA systems remain largely unknown. However, they have been associated with protection against phage infection or stabilization of genomic regions (Pecota and Wood, 1996; Fraikin et al., 2020; Peltier et al., 2020). In addition, they also contribute to the virulence and persistence of pathogenic bacteria in vivo in infection models (Helaine et al., 2014; Lobato-Márquez et al., 2016; Agarwal et al., 2020).

There are seven known classes of TA systems depending on the nature of the antitoxin and its mode of action on the toxin, with the toxin always being a protein. In Type I systems, the antitoxin is a small anti-sense RNA that forms a duplex with the toxin's mRNA to inhibit toxin production (Brantl, 2012). Type III antitoxins are RNAs that inactivate the toxin by forming a complex (Blower et al., 2012). For type IV, the antitoxin suppresses the toxicity of the toxin by stabilizing its targets (Masuda et al., 2012), and Type V is represented by the GhoT-GhoS system, in which the antitoxin inhibits the toxin by specific cleavage of its mRNA (Wang et al., 2012). In the type VI SocAB system of Caulobacter crescentus (Aakre et al., 2013), the SocB toxin is responsible for the essentiality of the $c l p X$ and $c l p P$ genes in this bacterium, and the SocA antitoxin serves as an adaptor protein to address the SocB toxin to the ClpXP $\mathrm{AAA}^{+}$ protease. For the recently identified type VII, the antitoxin neutralizes the toxin through post-translational modification of the toxin such as phosphorylation or oligoAMPylation (Songailiene et al., 2020; Yu et al., 2020). The most characterized TA systems are type II systems (Xie et al., 2018). In this case, the antitoxin is a protein that interacts with the toxin to form a complex in which the toxin is inactive (Van Melderen, 2010). They generally are auto-repressor of their own transcription, most often in complex with the toxin (Fraikin et al., 2020).

TA systems present in $M$. tuberculosis genome are mostly type II TA systems, including at least 51 VapBC systems, 10 MazEF, 1 PemIK, 2 RelBE, 1 YefM/YoeB, 3 HigBA, and 2 ParDE family members, as well as several newly identified systems including PezAT, PhoAT-PhoH2 and MbcTA. Besides, the DarTG system is a hybrid typeII/IV system and MenTA3 a type VII (Cai et al., 2020; Yu et al., 2020). M. tuberculosis TA systems are generally located within regions of horizontal gene transfer together with genes involved in virulence, dormancy, regulation or cell signaling (Ramage et al., 2009; Sala et al., 2014; Wang et al., 2015), suggesting that they could also contribute to the success of $M$. tuberculosis as a human pathogen. A substantial number of $M$. tuberculosis toxins have been cloned and showed toxicity when expressed in E. coli or in mycobacteria (Ramage et al., 2009; Sala et al., 2014; Agarwal et al., 2018; Akarsu et al., 2019). Besides, transcription of several $M$. tuberculosis TA systems were shown to be induced under various stress conditions including drug exposure, hypoxia, heat-shock, DNA damages (Sala et al., 2014; Tiwari et al., 2015; Gupta et al., 2017; Agarwal et al., 2018), and gene deletion mutants $\Delta v a p C 22, \Delta v a p B C 3 / 4 / 11$ and $\Delta m a z F 3 / 6 / 9$ are strongly impaired in host infection (Tiwari et al., 2015; Agarwal et al., 2018; Deep et al., 2018; Agarwal et al., 2020). Even though transcriptional induction of TA systems does not necessarily reflect toxin activation (LeRoux et al., 2020), these data suggest that toxins could modulate bacterial growth depending on environmental conditions, and thus contribute to $M$. tuberculosis physiology and virulence (Sala et al., 2014). This also implies that their toxic activity must be tightly regulated in order not to be detrimental for bacterial survival. Since all the TA systems described so far in $M$. tuberculosis encode protein toxins and antitoxins, one of the main control mechanism that could enable a fast change of Toxin/Antitoxin ratios in response to changing cellular conditions is differential proteolysis (Jenal and HenggeAronis, 2003; Molière and Turgay, 2013).

\section{PROTEOLYTIC REGULATION OF TOXIN ANTITOXIN SYSTEMS}

In bacteria, protein turnover is mainly achieved by multi-subunit machines known as AAA + proteases and the proteasome. It has been proposed that under certain conditions, type II antitoxins are degraded by $\mathrm{AAA}^{+}$proteases Lon or $\mathrm{Clp}$, which could result in lifting the repression of the operon and activation of the toxin (Van Melderen et al., 1994; Jensen and Gerdes, 1995; Koga et al., 2011). Except for the recently described degradation of a ParElike antitoxin of Microcystis aeruginosa PCC 7806 by a caspase homolog protease (Klemenčič et al., 2021), only $\mathrm{AAA}^{+}$proteases (ClpAP, ClpCP, ClpXP, Lon) have been involved in antitoxin degradation in bacteria (Van Melderen et al., 1994; Lehnherr and Yarmolinsky, 1995; Aizenman et al., 1996; Prysak et al., 2009; Donegan et al., 2010; Diago-Navarro et al., 2013; Muthuramalingam et al., 2016; Dubiel et al., 2018; Zhou et al., 2021).

How antitoxins are targeted to degradation remains largely unknown. Some appeared to be more susceptible to proteases due to their hydrophobic or flexible C-termini or to the presence of intrinsically disordered central regions (Yamaguchi et al., 2011). In some cases, antitoxin degradation might be assisted by specific adaptors, as it is the case for the Staphylococcus aureus adaptor protein TrfA that assists ClpCP-mediated degradation of the MazE antitoxin (Donegan et al., 2014), or even modulated by DNA (Dubiel et al., 2018; LeRoux et al., 2020). Although antitoxins are generally more sensitive to proteolysis than their cognate toxins, it is not known whether an antitoxin within a preformed TA complex can be directly targeted by proteases to induce toxin activation in vivo. Although it was suggested in vitro that an excess of the Lon protease could disrupt a preformed DinJ-YafQ complex in vitro (Ruangprasert et al., 2017), there is significant evidence showing that once a stable TA complex is formed, the antitoxin is generally protected from degradation (Dubiel et al., 2018; LeRoux et al., 2020; Lunge et al., 2020). Other attractive possibilities would be that certain stress conditions or alternative factors such as adaptors or chaperones would trigger TA complex dissociation in order to proteases to get access to their substrate antitoxin. In addition, cross-talks between multiple endogenous antitoxins from the same family (as found in $M$. tuberculosis) could also be involved in TA 
complex unstability. Indeed, non-cognate interactions between TA systems could lead to the formation of less stable non-cognate complexes with increased sensitivity to proteases, and potentially affect the promoter binding activities of TA complexes. Intriguingly, Leroux and colleagues (2020) recently showed for several chromosomal TA systems of $E$. coli that antitoxin degradation by different stresses led to the transcriptional derepression of their TA operon but in contrast, did not induce in any detectable toxin activation, thus further raising questions about how toxins can be activated and what is the role played by proteases in this process.

\section{MYCOBACTERIAL AAA ${ }^{+}$PROTEASES}

In M. tuberculosis, two cytosolic AAA + proteases have been identified: ClpC1P1P2 and ClpXP1P2. AAA + proteases combine a central ring-shaped peptidase $\mathrm{ClpP}$, together with a regulatory hexameric ring-shaped unfoldase $(\mathrm{ClpX}$ or $\mathrm{ClpC} 1)$ to bind and translocate the substrate to the central pore of the peptidase (Sauer and Baker, 2011). M. tuberculosis is one of the few bacteria that possess two essential $c l p P$ genes, which encode a heterooligomeric peptidase from a pair of homo-heptameric rings ClpP1P2 (Leodolter et al., 2015; Alhuwaider and Dougan, 2017; Vahidi et al., 2020). Interestingly, ClpX and ClpC1 unfoldases only interact with the ClpP2 ring surface (Leodolter et al., 2015). M. tuberculosis also encodes the membrane-bound $\mathrm{AAA}+$ protease $\mathrm{FtsH}$ that harbours peptidase and unfoldase activities on one single polypeptide. Apart from the fact that it can functionally complement some activities of $E$. coli FtsH (Srinivasan et al., 2006), its function in M. tuberculosis is poorly understood and transposon saturated mutagenesis did not firmly established its essentiality (Sassetti et al., 2003; DeJesus et al., 2017).

The Clp proteases of $M$. tuberculosis have been shown to be induced by stress conditions such as starvation or streptomycin exposure (Gupta et al., 2017). Moreover, clpC1 and $c l p P 1 P 2$ expression is directly activated under stress conditions by the regulator $\mathrm{ClgR}$, a stress regulator essential during macrophage infection and the reaeration response (Estorninho et al., 2010; Sherrid et al., 2010). Noticeably, ClgR is itself a substrate for ClpP1P2 proteolytic activity, indicating that $c l p$ genes regulation is tightly controlled in M. tuberculosis (Sherrid et al., 2010; Yamada and Dick, 2017). Both AAA + unfoldases ClpX and $\mathrm{ClpC1}$ are essential for the growth of M. tuberculosis $\mathrm{H} 37 \mathrm{Rv}$ (DeJesus et al., 2017; Lunge et al., 2020; Kester et al., 2021). ClpX has been shown to be involved in DNA replication and in cell division in M. tuberculosis (Dziedzic et al., 2010; Kester et al., 2021), and a global protein expression profiling following clpC1 gene silencing in $M$. tuberculosis showed that ClpC1P1P2 acts on several essential proteins involved in central metabolism and cell wall biosynthesis (Lunge et al., 2020). Similar to the ClpC1 homologue ClpA in E. coli, a small subset of ClpC1-sensitive proteins harbour typical $\mathrm{N}$-end degrons composed of four residues (Tyr, Phe, Trp, and Leu) known to be recognized by the ClpS adaptor in E. coli (Erbse et al., 2006). However, the vast majority of ClpC1P1P2-regulated proteins in M. tuberculosis have disorder-promoting residues (Pro, Arg, Gly, Gln, Ser, Glu, Lys, and Ala) within their terminal 15-aa region, and it was demonstrated that this is a critical feature for ClpC1P1P2 degradation of the small heat shock protein Hsp20 in $M$. tuberculosis (Lunge et al., 2020). ClpC1 recognition can also rely on the phosphorylation of an internal residue as shown for the anti-sigma factor RseA (Barik et al., 2010).

Depletion or drug-dependent inhibition of ClpP1P2 in $M$. tuberculosis identified four protein clients with putative degrons, namely ClgR, tmRNA SsrA and the two regulators WhiB1 and CarD, all four degradation signals located at the C-terminus and enriched in hydrophobic residues (Raju et al., 2012; Yamada and Dick, 2017). Comparison of their C-terminal with known E. coli ClpX subtrates (Flynn et al., 2003) suggests that these substrates (with the exception of WhiB1) might be recognized by ClpX (Alhuwaider and Dougan, 2017). Other studies revealed that the membrane-associated anti- $\sigma$ factor RsdA of M. tuberculosis was a ClpXP1P2 substrate and its Val-Ala-Ala internal degron was similar to the SsrA-tag (Jaiswal et al., 2013). More recently, similar degron sequences were identified in the cytoplasmic sequence of three other anti- $\sigma$ factors of $M$. tuberculosis but instead of leading to proteolysis, they affect the unfoldase activity of ClpX to regulate the inactive $\sigma /$ anti- $\sigma$ complex and thus modulate gene expression (Joshi et al., 2019). This is reminiscent of ClpX interaction with FtsZ that does not lead to altered intracellular levels of FtsZ but rather to an inhibition of Z-ring assembly in M. tuberculosis (Dziedzic et al., 2010).

Interestingly, $\mathrm{ClpC} 1$ is the target of antimycobacterial peptides such as cyclomarin A or lassomycin, and has emerged as an promising drug target (Lupoli et al., 2018; Fraga et al., 2019; Maurer et al., 2019). More generally, the activation, repression of modification of ClpP mechanism of action has been the focus of many studies to identify new antibiotics (Ye et al., 2016; MorenoCinos et al., 2019). For instance, acyldepsipeptides (ADEPs) kill M. tuberculosis by preventing the binding of AAA + regulatory unfoldases to ClpP1P2 (Famulla et al., 2016), peptide boronates prevent growth of $M$. tuberculosis by inhibition of ClpP1P2 active sites (Akopian et al., 2015) and pyrazinamide prodrug triggers ClpC1P1P2 dependent degradation of the essential PanD protein by modifying its oligomeric state (Gopal et al., 2020). To date, no adaptor protein has been described for M. tuberculosis ClpX (Alhuwaider and Dougan, 2017), although the essential DNA maintenance protein Single-Stranded DNA Binding protein (SSB) is able to activate ClpXP1P2 proteolytic activities (Kester et al., 2021). The only adaptor described so far in mycobacteria, ClpS, inhibits ClpC1-dependent unfolding and degradation of substrate SsrA, but also enhances the degradation of an $\mathrm{N}$-end rule model substrate in vitro (Marsee et al., 2018; Ziemski et al., 2020).

\section{INTERPLAY BETWEEN AAA ${ }^{+}$PROTEASES AND TOXIN ANTITOXIN SYSTEMS}

Several M. tuberculosis antitoxins have been demonstrated as ClpXP1P2 or ClpC1P1P2 substrates. One of the first proteomic study of ClpP1P2-dependent protein substrates in M. tuberculosis 


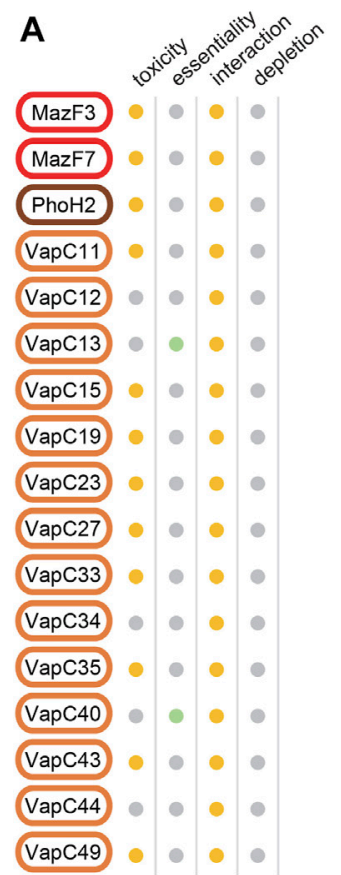

C

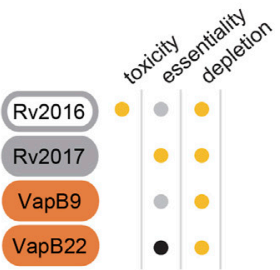

B
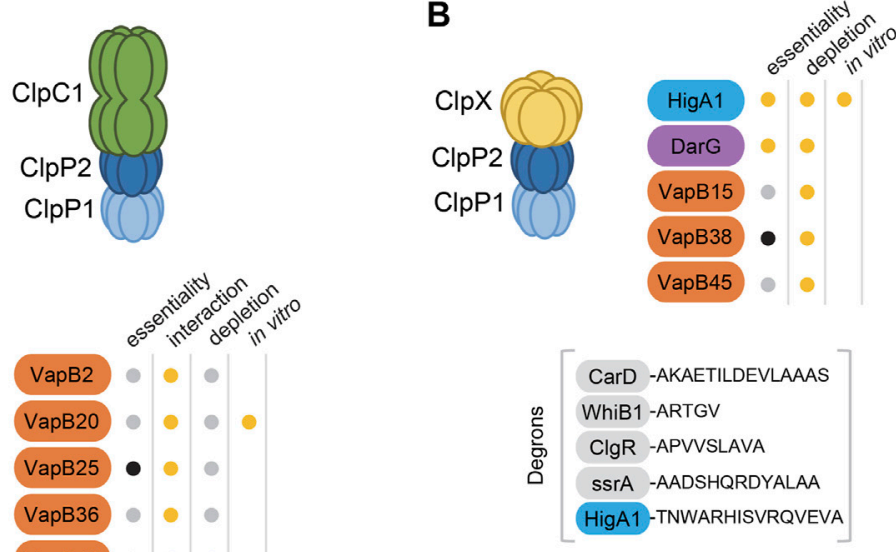

D

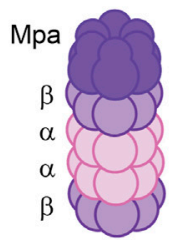

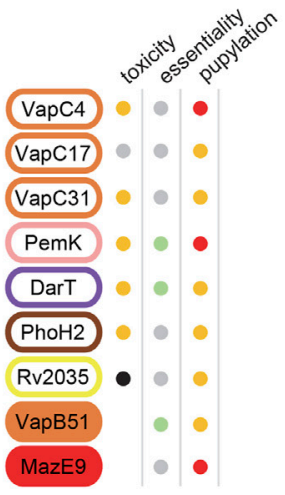

FIGURE 1 | Proteolytic regulation and recognition of Toxin-Antitoxin systems in $M$. tuberculosis. TA families are indicated by different colors as followed: Orange for VapBC, Green for ParDE, pink for PemlK, red for MazEF, dark blue for RelBE, purple for DarTG, bright blue for HigBA, brown for PhoAT-H2, yellow for ArsR-COG3832 and grey for unknown. Toxins and Antitoxins are indicated by filled and open rounded rectangles, respectively. Toxin and Antitoxin proteins are candidate substrates for proteases, (A) ClpC1P1P2 (B) ClpXP1P2, (C) ClpP1P2 (the associated chaperone subunit, either ClpX or ClpC1 is to be determined), (D) the Mpa-proteasome. Known degrons for ClpXP1P2 are indicated in (B) under brackets. Functional properties were indicated in the columns adjacent to the toxins and antitoxins, i.e., toxicity, essentiality (essential), interaction, accumulation following protease depletion (depletion), in vitro degradation (in vitro) or pupylation. Toxicity: orange dots mean toxic when overexpressed (Ramage et al., 2009; Sala et al., 2014; Agarwal et al., 2018; Akarsu et al., 2019) in at least one bacterial host (M. tuberculosis, M. smegmatis or E. coli), grey dots nontoxic and black dot non tested. Essentiality (DeJesus et al., 2017): orange dots mean essential, green dots mean growth advantage when mutated, grey dots non-essential, black dots mean uncertain or non-tested. Interaction with chaperone subunit ClpC1 in vivo (Ziemski et al., 2020): orange dots mean interaction and grey dots no interaction. Depletion of clpP1P2, clpP2 or clpC1 (Raju et al., 2014; Lunge et al., 2020): orange dots mean protein stabilization and grey dots mean no detectable protein changes. In vitro degradation assays (Ziemski et al., 2020; Texier et al., 2021): orange dots mean degradation. Pupylation: orange dots mean pupylated under routine culture conditions (Festa et al., 2010), red dots mean pupylated by reconstituted system in E. coli and/or in vitro (Chi et al., 2018).

following depletion of endogenous ClpP1P2 identified 6 antitoxins as putative ClpP1P2 substrates, namely MazE10, VapB22, VapB9, VapB41, Rv2017 and HigA1 (Raju et al., 2014); Figure 1). Among these antitoxins, MazE10 was later identified as a likely ClpC1P1P2 substrate in vivo, together with VapB47 (Lunge et al., 2020); Figure 1). Note that both MazE10 and VapB47 antitoxins have disorder-promoting residues at their C-terminal end, which was suggested to be important for ClpC1 recognition (Lunge et al., 2020). It is striking that when we applied similar search for disordered C-terminal ends, we found that more than $60 \%$ of the antitoxins of $M$. tuberculosis possess this type of $\mathrm{C}$-terminal region, thus suggesting that disordered C-terminal ends could indeed contribute to recognition by proteases. Note that a table presenting the C-terminal ends of all known M. tuberculosis antitoxins can be found in Texier and colleagues (Texier et al., 2021). The HigA1 antitoxin possesses a typical C-terminal ClpX degron with two crucial hydrophobic last residues Val-Ala that were recently 
shown to be the recognition sequence of HigA1 by ClpX in $M$. tuberculosis (Texier et al., 2021). Noticeably, the Rv2017 antitoxin contains a ClpX-like degron with two hydrophobic residues located at its extreme C-terminal part (Ala-Ile), suggesting that it could also be recognized by ClpX. The last four ClpP1P2dependent antitoxins, namely VapB9, VapB22, VapB41 harbor different C-terminal ends and no other common feature could be detected (Raju et al., 2014). This suggests that the ClpXP1P2 and ClpC1P1P2-dependent degradation signals are not restricted to typical degrons, and that their degradation might rely on posttranslational modifications or on unknown adaptors (Trentini et al., 2016).

In a recent CRISPRi study performed in M. tuberculosis, 4 antitoxins, namely VapB15, VapB38, VapB45 and DarG, were found to be up-regulated upon ClpP2 depletion but not upon ClpC1 depletion in M. tuberculosis (Lunge et al., 2020), suggesting that these antitoxins might be ClpXP1P2 substrates. This is particularly likely for VapB45, which contains a typical ClpXdegron at its C-terminus (Ala-Ala). A systematic search for $\mathrm{ClpC1}$ interactors based on the bacterial adenylate cyclase two-hybrid (BACTH) screen in E. coli showed that type II TA systems of $M$. tuberculosis are one of the largest group of ClpC1 interacting partners (Ziemski et al., 2020). Members of the VapBC, MazEF and ParDE TA families were identified, with VapBC systems being the most abundant pairs found to be interacting partners of the ClpC1P1P2 protease complex (20 out of the 51 known VapBC pairs; Figure 1). Both VapB20 and the RelB1 antitoxins were further confirmed to be specific substrates for ClpC1P1P2 and not ClpXP1P2 using in vitro degradation assays (Ziemski et al., 2020), thus suggesting that $\mathrm{ClpC} 1$ and $\mathrm{ClpX}$ chaperones may not share substrate recognition motifs. Note that VapC20 or RelE1 toxin form stable complexes with their cognate antitoxin in which the antitoxin is protected from degradation, further raising questions about how the toxin could be freed from the antitoxin in order to be activated (see above comments).

Interestingly, the HigA1 degron has been the only ClpXdependent recognition sequence identified so far for a mycobacterial antitoxin (Texier et al., 2021). HigA1 is part of the tripartite toxin-antitoxin-chaperone (TAC) system of $M$. tuberculosis that includes a cognate SecB-like chaperone $\left(\mathrm{SecB}^{\mathrm{TA}}\right)$. In most Gram-negative bacteria, SecB targets presecretory proteins to the Sec translocon located at the inner membrane (Bechtluft et al., 2010). In contrast with classical two-component TA systems, the TAC toxinantitoxin pair is tightly controlled by $\mathrm{SecB}^{\mathrm{TA}}$, through a direct interaction between the chaperone and an unusual aggregation-prone $\mathrm{C}$-terminal extension of the antitoxin HigA1, named ChAD (chaperone-addiction) (Bordes et al., 2011; Bordes et al., 2016; Guillet et al., 2019). Binding of $\mathrm{SecB}^{\mathrm{TA}}$ to the $\mathrm{ChAD}$ of the antitoxin protects HigAl from aggregation and degradation. Remarkably, both $\mathrm{SecB}^{\mathrm{TA}}$ binding site and ClpX degron are located within the same ChAD region of the antitoxin (with different residues being involved). These data suggest that under certain stress conditions, $\mathrm{SecB}^{\mathrm{TA}}$ could be hijacked by protein substrates (either aggregated pre-proteins or specific exported proteins) and the HigA1 antitoxin could be degraded by ClpXP1P2, which could lead to a transient activation of the HigB1 toxin until normal growth conditions resume.

The analysis of the amino acid sequence of $M$. tuberculosis antitoxins suggests that only VapB19, VapB41, VapB44, VapB45, Rv1990c, Rv2017 and HigA2 antitoxins possess a putative HigA1like degron sequence with at least two hydrophobic residues at their extreme C-terminus (respectively, Leu-Ala, Ala-Ala-Leu, Ala-Val, Ala-Ile-Ala-Ala, Val-Phe-Val, Ala-Ile and Leu-Ala). These hydrophobic residues are mainly non-polar aliphatic (Ala, Val, Leu, Ile) as usually observed in C-terminal ClpX degrons, except for Rv1990c that presents an aromatic phenylalanine. In addition, VapB41, VapB44, VapB45 and Rv1990c also possess an acidic residue before the hydrophobic end, as found in HigA1. This suggests that these antitoxins could also be recognized by $M$. tuberculosis ClpX. In addition, five poorly conserved VapB antitoxins (VapB19, VapB23, VapB28, VapB30 and VapB34) share a highly similar extreme C-terminus, with hydrophobic residues following an arginine (Arg-Gly-LeuPro-Ala-Pro, Arg-Gly-Leu-Pro-Ala or Arg-Leu-Gly-Leu-Ala motifs), suggesting that these antitoxins could share similar degrons (Texier et al., 2021).

Remarkably, toxins were also identified as proteases targets or putative substrates. Indeed, while only $6 \mathrm{VapB}$ antitoxins were identified as $\mathrm{ClpC1}$ interactors in vivo, the remaining 14 interactors were VapC toxins (Lunge et al., 2020). These intriguing results suggest that toxins might themselves be the targets of proteases or in contrary, act as bona fide protease adaptors for their cognate antitoxins and thus being actor of their own activation. Moreover, the degradation of the toxin could also be part of a bacterial strategy to resume growth after TA system activation, as demonstrated for the type I toxin HokB in E. coli (Wilmaerts et al., 2019). In this case, awakening of HokB-induced persister cells was shown to require the degradation of HokB monomers by the periplasmic stress protease DegQ. Whether such mechanism exists in $M$. tuberculosis remains to be determined.

\section{POSSIBLE LINK BETWEEN TOXIN ANTITOXIN AND THE PUP-PROTEASOME SYSTEM}

Another peculiarity of Actinomycetes is to possess a eukaryoticlike proteasome (Festa et al., 2010; Müller and Weber-Ban, 2019). The mycobacterial proteasome consists of a highly conserved central peptidase core particle (20S CP) composed of 28 subunits ( 2 heptameric inner rings composed of PcrB subunits, and 2 heptameric outer rings composed of PcrA subunits), which is gated and interact with ring-shaped activators to form a fully active protease capable of degrading specific sets of cellular substrates (Figure 1). With the mycobacterial proteasomal $\mathrm{AAA}+\mathrm{Mpa}$, the proteasome targets substrates that have been post-translationally modified with Pup (prokaryotic ubiquitinlike protein) by a dedicated ligase PafA (Pearce et al., 2008; Burns et al., 2009). Hundreds of M. tuberculosis pupylated proteins, which include Mpa and PafA, have been identified by proteomics 
studies, even though many of them are not degraded under normal growth conditions (Festa et al., 2010; Müller and Weber-Ban, 2019). This could be reminiscent of the Pup degradation-independent regulatory role in several bacterial species (Elharar et al., 2014; Küberl et al., 2016).

In mycobacteria, Pup goes through a deamidation step by the Dop enzyme before it can be attached to a target by PafA, and Pup can also be removed from tagged substrates by Dop, as well or transferred between substrates by PafA (Burns et al., 2010; Imkamp et al., 2010; Zhang et al., 2017). These enzymatic activities must be tightly regulated in order to avoid useless cycles of pupylation/depupylation and even though little is known about these regulations, it was shown that Pup-free Dop is depleted under stress conditions leading to accelerated proteasomal degradation (Elharar et al., 2016) and that the AAA + protease ClpC1P1P2 is responsible for the depletion of Pup-free Dop under starvation conditions (Hecht et al., 2020). Dop, PafA, Pup, Mpa, and 20S CPs constitute the core "Pup-proteasome system" (PPS). Two other partners of the 20S CP have been described in M. tuberculosis: The non-ATPase activator Bpa (also known as PafE) could address unstructured substrates to proteasomal degradation (Delley et al., 2014; Jastrab et al., 2015), and the AAA + Cpa (a Cdc48-like protein), that interacts with the $20 \mathrm{~S}$ core but for which no degradation substrate has been identified yet (Ziemski et al., 2018). PPS mutants of $M$. tuberculosis are viable but are highly attenuated in a mouse infection model (Darwin et al., 2003; Gandotra et al., 2007), are highly sensitive to NO due to the failure to degrade a single pupylated substrate, Log (MacMicking et al., 1997; Samanovic et al., 2015) and are unable to use nitrate as a nitrogen source (Becker et al., 2019). Moreover, a bpa mutant displays a slow growth in vitro and in mice and is hypersensitive to heat shock (Jastrab et al., 2015). Anti-TB drugs targeting the mycobacterial proteasome are promising but they face the challenge of being highly selective in order not to inhibit the human proteasome (Lin et al., 2013; Bibo-Verdugo et al., 2017; Zhan et al., 2019).

Although $\mathrm{AAA}^{+}$proteases are the main proteases shown to be involved in antitoxin degradation (Muthuramalingam et al., 2016), several studies suggest that the PPS could also be involved in the regulation of TA systems. Indeed five toxins, namely Rv2035, DarT, PhoH2, VapC17 and VapC31, and the VapB51 antitoxin are part of the $M$. tuberculosis pupylome under standard laboratory growth conditions culture conditions (Festa et al., 2010). Note that it remains to be determined whether these proteins are directly pupylated and if pupylation leads to their degradation by the PPS. In addition, the reconstitution of a pupylation system in E. coli and in vitro showed that the VapC4 and PemK toxins, and the MazE9 antitoxin could also be pupylated (Chi et al., 2018). Intriguingly, $\mathrm{PhoH} 2$ is the only toxin potentially regulated both by the proteasome and ClpC1P1P2 (Figure 1), possibly to ensure low toxin level. The fact that there are more potentially pupylated toxins than antitoxins is striking and suggests that some toxins might be differently regulated by proteasomal degradation and antitoxin inhibition (Burns et al., 2010). Yet, the M. tuberculosis pupylome was performed under standard laboratory growth conditions and we cannot exclude that more antitoxins could be pupylated under certain stresses, as growth conditions were shown to modify the abundance of pupylated proteins (Becker et al., 2019). The fact that Bpa could drive proteasomal degradation of partially or totally unfolded proteins, which is a property of many antitoxins, suggests that other proteasome activators could be involved in TA proteins turnover (Jastrab et al., 2015). Finally, there could also be a link between the master regulator PafBC, which is encoded within the PPS gene locus and the regulation of TA systems, as the VapB antitoxin of $M$. smegmatis was shown to be part of the pafBC regulon (Müller et al., 2018).

\section{CONCLUDING REMARKS}

A substantial number of $M$. tuberculosis antitoxins are bona fide substrates of $\mathrm{AAA}^{+}$proteases, both in vivo and in vitro (Figure 1). Yet, there is very little knowledge about recognition signals within antitoxins and degron sequences are just beginning to emerge. In addition, it is not known whether proteases directly play a role in toxin activation in vivo and, if they do, at which stage of the TA activation cycle such a regulation would occur (Sala et al., 2017; LeRoux et al., 2020). Similarly, it remains to be determined whether or not the control of TA systems by proteolysis relies on a specific activation or induction of proteases (Ramisetty, 2020). Moreover, there is a clear lack of data concerning additional factors such as stress-induced protease adaptors and chaperones, or specific environmental stimuli (or perhaps cell cycle events and other host factors) that might trigger antitoxin degradation and the subsequent toxin activation and/or expression of the TA operon.

Many toxins of $M$. tuberculosis have been cloned, overexpressed and shown to be toxic (Ramage et al., 2009; Sala et al., 2014; Agarwal et al., 2018; Akarsu et al., 2019). Remarkably, several of these toxins were capable of efficiently inducing cell death and their respective antitoxins were essential for M. tuberculosis growth (Fivian-Hughes and Davis, 2010; Schuessler et al., 2013; DeJesus et al., 2017; Freire et al., 2019; Cai et al., 2020; Zaveri et al., 2020). This suggests that proteolysis has to be tightly regulated in order to avoid unwanted proteolysis of antitoxins, which could be detrimental for $M$. tuberculosis growth. Yet, under certain conditions, a transient growth inhibition might be beneficial for the pathogen, especially for the entry into a persistent mode.

Finally, the fact that a significant number of toxins were shown to interact with proteases (both $\mathrm{ClpC} 1 / \mathrm{P} 1 \mathrm{P} 2$ and the proteasome) suggests that proteolysis could ensure that deleterious toxins do not accumulate. Most of these toxins are part of type 2 TA systems, suggesting that that under certain conditions, inhibition by their cognate antitoxins might not be robust enough without additional control of the toxin by proteolysis. Although new drug discovery strategies that focus on inhibiting mycobacterial proteases seem promising (Lupoli et al., 2018), it is important to note here that such a potentially dual role of proteases on toxin activation or inhibition in $M$. tuberculosis could lead to unwanted toxin activation and the subsequent entry into a persistent mode. Intriguingly, we also noticed that except 
for DarTG, none of the toxins and antitoxins that interact with proteases are part of the same TA pairs (Figure 1), suggesting a highly complex network of interactions and antagonistic effects that could impact growth of the pathogen in respond to specific signals. More work is needed to uncover such a complex reservoir of interactions involving highly conserved proteolysis pathways and the multiple stress-responsive TA systems of M. tuberculosis.

\section{AUTHOR CONTRIBUTIONS}

PB and PG wrote the paper.

\section{REFERENCES}

Aakre, C. D., Phung, T. N., Huang, D., and Laub, M. T. (2013). A Bacterial Toxin Inhibits DNA Replication Elongation through a Direct Interaction with the $\beta$ Sliding Clamp. Mol. Cel 52, 617-628. doi:10.1016/j.molcel.2013.10.014

Agarwal, S., Sharma, A., Bouzeyen, R., Deep, A., Sharma, H., Mangalaparthi, K. K., et al. (2020). VapBC22 Toxin-Antitoxin System from Mycobacterium Tuberculosis Is Required for Pathogenesis and Modulation of Host Immune Response. Sci. Adv. 6, eaba6944. doi:10.1126/sciadv.aba6944

Agarwal, S., Tiwari, P., Deep, A., Kidwai, S., Gupta, S., Thakur, K. G., et al. (2018). System-Wide Analysis Unravels the Differential Regulation and In Vivo Essentiality of Virulence-Associated Proteins B and C Toxin-Antitoxin Systems of Mycobacterium Tuberculosis. J. Infect. Dis. 217, 1809-1820. doi:10.1093/infdis/jiy109

Aizenman, E., Engelberg-Kulka, H., and Glaser, G. (1996). An Escherichia coli Chromosomal "Addiction Module"Regulated by Guanosine [corrected] 3',5'Bispyrophosphate: a Model for Programmed Bacterial Cell Death. Proc. Natl. Acad. Sci. 93, 6059-6063. doi:10.1073/pnas.93.12.6059

Akarsu, H., Bordes, P., Mansour, M., Bigot, D.-J., Genevaux, P., and Falquet, L. (2019). TASmania: A Bacterial Toxin-Antitoxin Systems Database. PLoS Comput. Biol. 15, e1006946. doi:10.1371/journal.pcbi.1006946

Akopian, T., Kandror, O., Tsu, C., Lai, J. H., Wu, W., Liu, Y., et al. (2015). Cleavage Specificity of Mycobacterium tuberculosis ClpP1P2 Protease and Identification of Novel Peptide Substrates and Boronate Inhibitors with Anti-bacterial Activity. J. Biol. Chem. 290, 11008-11020. doi:10.1074/jbc.M114.625640

Alhuwaider, A. A. H., and Dougan, D. A. (2017). AAA+ Machines of Protein Destruction in Mycobacteria. Front. Mol. Biosci. 4, 49. doi:10.3389/fmolb.2017.00049

Barik, S., Sureka, K., Mukherjee, P., Basu, J., and Kundu, M. (2010). RseA, The SigE Specific Anti-Sigma Factor of Mycobacterium Tuberculosis, Is Inactivated by Phosphorylation-Dependent ClpC1P2 Proteolysis. Mol. Microbiol. 75, 592-606. doi:10.1111/j.1365-2958.2009.07008.x

Bechtluft, P., Nouwen, N., Tans, S. J., and Driessen, A. J. M. (2010). SecB-A Chaperone Dedicated to Protein Translocation. Mol. BioSyst. 6, 620-627. doi:10.1039/b915435c

Becker, S. H., Jastrab, J. B., Dhabaria, A., Chaton, C. T., Rush, J. S., Korotkov, K. V., et al. (2019). TheMycobacterium Tuberculosis Pup-Proteasome System Regulates Nitrate Metabolism Through an Essential Protein Quality Control Pathway. Proc. Natl. Acad. Sci. USA 116, 3202-3210. doi:10.1073/pnas. 1819468116

Bibo-Verdugo, B., Jiang, Z., Caffrey, C. R., and O’Donoghue, A. J. (2017). Targeting Proteasomes in Infectious Organisms to Combat Disease. FEBS J. 284, 1503-1517. doi:10.1111/febs.14029

Blower, T. R., Short, F. L., Rao, F., Mizuguchi, K., Pei, X. Y., Fineran, P. C., et al. (2012). Identification and Classification of Bacterial Type III Toxin-Antitoxin Systems Encoded in Chromosomal and Plasmid Genomes. Nucleic Acids Res. 40, 6158-6173. doi:10.1093/nar/gks231

Bordes, P., Cirinesi, A.-M., Ummels, R., Sala, A., Sakr, S., Bitter, W., et al. (2011). SecB-like Chaperone Controls a Toxin-Antitoxin Stress-Responsive System in Mycobacterium tuberculosis. Proc. Natl. Acad. Sci. 108, 8438-8443. doi:10. 1073/pnas.1101189108

\section{FUNDING}

Agence Nationale de la Recherche (ANR-13-BSV8-0010-01 and ANR-19-CE12-0026), CNRS basic support.

\section{ACKNOWLEDGMENTS}

The authors are grateful to Pauline Texier and David Dougan for fruitful discussion. This work was supported by the Agence Nationale de la Recherche (ANR-13-BSV8-0010-01 and ANR19-CE12-0026).

Bordes, P., Sala, A. J., Ayala, S., Texier, P., Slama, N., Cirinesi, A.-M., et al. (2016). Chaperone Addiction of Toxin-Antitoxin Systems. Nat. Commun. 7, 13339. doi:10.1038/ncomms13339

Brantl, S. (2012). Bacterial Type I Toxin-Antitoxin Systems. RNA Biol. 9, 1488-1490. doi:10.4161/rna.23045

Burns, K. E., Cerda-Maira, F. A., Wang, T., Li, H., Bishai, W. R., and Darwin, K. H. (2010). "Depupylation" of Prokaryotic Ubiquitin-Like Protein From Mycobacterial Proteasome Substrates. Mol. Cel 39, 821-827. doi:10.1016/j. molcel.2010.07.019

Burns, K. E., Liu, W.-T., Boshoff, H. I. M., Dorrestein, P. C., and Barry, C. E. (2009). Proteasomal Protein Degradation in Mycobacteria Is Dependent Upon a Prokaryotic Ubiquitin-Like Protein. J. Biol. Chem. 284, 3069-3075. doi:10. 1074/jbc.M808032200

Cai, Y., Usher, B., Gutierrez, C., Tolcan, A., Mansour, M., Fineran, P. C., et al. (2020). A Nucleotidyltransferase Toxin Inhibits Growth of Mycobacterium Tuberculosis Through Inactivation of tRNA Acceptor Stems. Sci. Adv. 6, eabb6651. doi:10.1126/sciadv.abb6651

Chi, X., Chang, Y., Li, M., Lin, J., Liu, Y., Li, C., et al. (2018). Biochemical Characterization of mt-Pem Ik, A Novel Toxin-antitoxin System in Mycobacterium Tuberculosis. FEBS Lett. 592, 4039-4050. doi:10.1002/18733468.13280

Darwin, K. H., Ehrt, S., Gutierrez-Ramos, J.-C., Weich, N., and Nathan, C. F. (2003). The Proteasome of Mycobacterium tuberculosis Is Required for Resistance to Nitric Oxide. Science 302, 1963-1966. doi:10.1126/science. 1091176

Deep, A., Tiwari, P., Agarwal, S., Kaundal, S., Kidwai, S., Singh, R., et al. (2018) Structural, Functional and Biological Insights Into the Role of Mycobacterium Tuberculosis VapBC11 Toxin-Antitoxin System: Targeting a tRNase to Tackle Mycobacterial Adaptation. Nucleic Acids Res. 46, 11639-11655. doi:10.1093/ nar/gky924

DeJesus, M. A., Gerrick, E. R., Xu, W., Park, S. W., Long, J. E., Boutte, C. C., et al. (2017). Comprehensive Essentiality Analysis of the Mycobacterium Tuberculosis Genome Via Saturating Transposon Mutagenesis. mBio 8, e02133. doi:10.1128/mBio.02133-16

Delley, C. L., Laederach, J., Ziemski, M., Bolten, M., Boehringer, D., and WeberBan, E. (2014). Bacterial Proteasome Activator Bpa (Rv3780) Is a Novel RingShaped Interactor of the Mycobacterial Proteasome. PLoS One 9, e114348. doi:10.1371/journal.pone.0114348

Diago-Navarro, E., Hernández-Arriaga, A. M., Kubik, S., Konieczny, I., and DíazOrejas, R. (2013). Cleavage of the Antitoxin of the parD Toxin-Antitoxin System Is Determined by the ClpAP Protease and Is Modulated by the Relative Ratio of the Toxin and the Antitoxin. Plasmid 70, 78-85. doi:10.1016/j.plasmid. 2013.01.010

Donegan, N. P., Marvin, J. S., and Cheung, A. L. (2014). Role of Adaptor TrfA and ClpPC in Controlling Levels of SsrA-Tagged Proteins and Antitoxins in Staphylococcus Aureus. J. Bacteriol. 196, 4140-4151. doi:10.1128/JB.02222-14

Donegan, N. P., Thompson, E. T., Fu, Z., and Cheung, A. L. (2010). Proteolytic Regulation of Toxin-Antitoxin Systems by ClpPC in Staphylococcus Aureus. J. Bacteriol. 192, 1416-1422. doi:10.1128/JB.00233-09

Dubiel, A., Wegrzyn, K., Kupinski, A. P., and Konieczny, I. (2018). ClpAP Protease Is a Universal Factor that Activates the parDE Toxin-Antitoxin System From a 
Broad Host Range RK2 Plasmid. Sci. Rep. 8, 15287. doi:10.1038/s41598-01833726-y

Dziedzic, R., Kiran, M., Plocinski, P., Ziolkiewicz, M., Brzostek, A., Moomey, M., et al. (2010). Mycobacterium Tuberculosis ClpX Interacts with FtsZ and Interferes with FtsZ Assembly. PLoS One 5, e11058. doi:10.1371/journal. pone. 0011058

Elharar, Y., Roth, Z., Hecht, N., Rotkopf, R., Khalaila, I., and Gur, E. (2016). Posttranslational Regulation of Coordinated Enzyme Activities in the PupProteasome System. Proc. Natl. Acad. Sci. USA 113, E1605-E1614. doi:10.1073/ pnas. 1525185113

Elharar, Y., Roth, Z., Hermelin, I., Moon, A., Peretz, G., Shenkerman, Y., et al. (2014). Survival of Mycobacteria Depends on Proteasome-mediated Amino Acid Recycling under Nutrient Limitation. EMBO J. 33, 1802-1814. doi:10. 15252/embj.201387076

Erbse, A., Schmidt, R., Bornemann, T., Schneider-Mergener, J., Mogk, A., Zahn, R., et al. (2006). ClpS Is an Essential Component of the N-End Rule Pathway in Escherichia Coli. Nature 439, 753-756. doi:10.1038/nature04412

Estorninho, M., Smith, H., Thole, J., Harders-Westerveen, J., Kierzek, A., Butler, R. E., et al. (2010). ClgR Regulation of Chaperone and Protease Systems Is Essential for Mycobacterium Tuberculosis Parasitism of the Macrophage. Microbiology (Reading) 156, 3445-3455. doi:10.1099/mic.0.042275-0

Famulla, K., Sass, P., Malik, I., Akopian, T., Kandror, O., Alber, M., et al. (2016). Acyldepsipeptide Antibiotics Kill Mycobacteria by Preventing the Physiological Functions of the ClpP1P2 Protease. Mol. Microbiol. 101, 194-209. doi:10.1111/ mmi. 13362

Festa, R. A., McAllister, F., Pearce, M. J., Mintseris, J., Burns, K. E., Gygi, S. P., et al. (2010). Prokayrotic Ubiquitin-Like Protein (Pup) Proteome of Mycobacterium Tuberculosis. PLoS ONE 5, e8589. doi:10.1371/journal.pone.0008589

Fivian-Hughes, A. S., and Davis, E. O. (2010). Analyzing the Regulatory Role of the HigA Antitoxin within Mycobacterium Tuberculosis. J. Bacteriol. 192, 4348-4356. doi:10.1128/jb.00454-10

Flynn, J. M., Neher, S. B., Kim, Y.-I., Sauer, R. T., and Baker, T. A. (2003). Proteomic Discovery of Cellular Substrates of the ClpXP Protease Reveals Five Classes of ClpX-Recognition Signals. Mol. Cel 11, 671-683. doi:10.1016/s10972765(03)00060-1

Fraga, H., Rodriguez, B., Bardera, A., Cid, C., Akopian, T., Kandror, O., et al. (2019). Development of High Throughput Screening Methods for Inhibitors of ClpC1P1P2 from Mycobacteria Tuberculosis. Anal. Biochem. 567, 30-37. doi:10.1016/j.ab.2018.12.004

Fraikin, N., Goormaghtigh, F., and Van Melderen, L. (2020). Type II ToxinAntitoxin Systems: Evolution and Revolutions. J. Bacteriol. 202, 19. doi:10. 1128/JB.00763-19

Freire, D. M., Gutierrez, C., Garza-Garcia, A., Grabowska, A. D., Sala, A. J., Ariyachaokun, K., et al. (2019). An NAD+ Phosphorylase Toxin Triggers Mycobacterium Tuberculosis Cell Death. Mol. Cel 73, 1282-1291. doi:10. 1016/j.molcel.2019.01.028

Gandotra, S., Schnappinger, D., Monteleone, M., Hillen, W., and Ehrt, S. (2007). In vivo Gene Silencing Identifies the Mycobacterium Tuberculosis Proteasome as Essential for the Bacteria to Persist in Mice. Nat. Med. 13, 1515-1520. doi:10. $1038 / \mathrm{nm} 1683$

Gopal, P., Sarathy, J. P., Yee, M., Ragunathan, P., Shin, J., Bhushan, S., et al. (2020). Pyrazinamide Triggers Degradation of its Target Aspartate Decarboxylase. Nat. Commun. 11, 1661. doi:10.1038/s41467-020-15516-1

Guillet, V., Bordes, P., Bon, C., Marcoux, J., Gervais, V., Sala, A. J., et al. (2019). Structural Insights into Chaperone Addiction of Toxin-Antitoxin Systems. Nat. Commun. 10, 782. doi:10.1038/s41467-019-08747-4

Gupta, A., Venkataraman, B., Vasudevan, M., and Gopinath Bankar, K. (2017). CoExpression Network Analysis of Toxin-Antitoxin Loci in Mycobacterium Tuberculosis Reveals Key Modulators of Cellular Stress. Sci. Rep. 7, 5868. doi:10.1038/s41598-017-06003-7

Harms, A., Brodersen, D. E., Mitarai, N., and Gerdes, K. (2018). Toxins, Targets, and Triggers: An Overview of Toxin-Antitoxin Biology. Mol. Cel 70, 768-784. doi:10.1016/j.molcel.2018.01.003

Hecht, N., Becher, M., Korman, M., Vishkautzan, M., and Gur, E. (2020). Interand Intramolecular Regulation of Protein Depupylation in Mycobacterium Smegmatis. Febs J. 287, 4389-4400. doi:10.1111/febs.15245

Helaine, S., Cheverton, A. M., Watson, K. G., Faure, L. M., Matthews, S. A., and Holden, D. W. (2014). Internalization of Salmonella by Macrophages Induces
Formation of Nonreplicating Persisters. Science 343, 204-208. doi:10.1126/ science. 1244705

Imkamp, F., Striebel, F., Sutter, M., Özcelik, D., Zimmermann, N., Sander, P., et al. (2010). Dop Functions as a Depupylase in the Prokaryotic Ubiquitin-Like Modification Pathway. EMBO Rep. 11, 791-797. doi:10.1038/embor.2010.119

Jaiswal, R. K., Prabha, T. S., Manjeera, G., and Gopal, B. (2013). Mycobacterium Tuberculosis RsdA Provides a Conformational Rationale for Selective Regulation of $\sigma$-factor Activity by Proteolysis. Nucleic Acids Res. 41, 3414-3423. doi:10.1093/nar/gks1468

Jastrab, J. B., Wang, T., Murphy, J. P., Bai, L., Hu, K., Merkx, R., et al. (2015). An Adenosine Triphosphate-independent Proteasome Activator Contributes to the Virulence of Mycobacterium Tuberculosis. Proc. Natl. Acad. Sci. U.S.A. 112, E1763-E1772. doi:10.1073/pnas.1423319112

Jenal, U., and Hengge-Aronis, R. (2003). Regulation by Proteolysis in Bacterial Cells. Curr. Opin. Microbiol. 6, 163-172. doi:10.1016/s1369-5274(03)00029-8

Jensen, R. B., and Gerdes, K. (1995). Programmed Cell Death in Bacteria: Proteic Plasmid Stabilization Systems. Mol. Microbiol. 17, 205-210. doi:10.1111/j.13652958.1995.mmi_17020205.x

Joshi, A. C., Kaur, P., Nair, R. K., Lele, D. S., Nandicoori, V. K., and Gopal, B. (2019). Selectivity Among Anti- $\sigma$ Factors by Mycobacterium Tuberculosis ClpX Influences Intracellular Levels of Extracytoplasmic Function $\sigma$ Factors. J. Bacteriol. 201, 18. doi:10.1128/JB.00748-18

Kester, J. C., Kandror, O., Akopian, T., Chase, M. R., Zhu, J., Rubin, E. J., et al. (2021). ClpX Is Essential and Activated by Single-Strand DNA Binding Protein in Mycobacteria. J. Bacteriol. 203, 20. doi:10.1128/JB.00608-20

Klemenčič, M., Halužan Vasle, A., and Dolinar, M. (2021). The Cysteine Protease $\mathrm{MaOC1}$, A Prokaryotic Caspase Homolog, Cleaves The Antitoxin of a Type II Toxin-Antitoxin System. Front. Microbiol. 12, 635684. doi:10.3389/fmicb.2021. 635684

Koga, M., Otsuka, Y., Lemire, S., and Yonesaki, T. (2011). Escherichia Coli rnlA and rnlB Compose A Novel Toxin-Antitoxin System. Genetics 187, 123-130. doi:10.1534/genetics.110.121798

Küberl, A., Polen, T., and Bott, M. (2016). The Pupylation Machinery Is Involved in Iron Homeostasis by Targeting the Iron Storage Protein Ferritin. Proc. Natl. Acad. Sci. USA 113, 4806-4811. doi:10.1073/pnas.1514529113

Lehnherr, H., and Yarmolinsky, M. B. (1995). Addiction Protein Phd of Plasmid Prophage P1 Is A Substrate of the ClpXP Serine Protease of Escherichia coli. Proc. Natl. Acad. Sci. 92, 3274-3277. doi:10.1073/pnas.92.8.3274

Leodolter, J., Warweg, J., and Weber-Ban, E. (2015). The Mycobacterium tuberculosis ClpP1P2 Protease Interacts Asymmetrically with Its ATPase Partners ClpX and ClpC1. PLoS One 10, e0125345. doi:10.1371/journal. pone. 0125345

LeRoux, M., Culviner, P. H., Liu, Y. J., Littlehale, M. L., and Laub, M. T. (2020). Stress Can Induce Transcription of Toxin-Antitoxin Systems Without Activating Toxin. Mol. Cel 79, 280-292. doi:10.1016/j.molcel.2020.05.028

Lin, G., Chidawanyika, T., Tsu, C., Warrier, T., Vaubourgeix, J., Blackburn, C., et al. (2013). N,C-Capped Dipeptides with Selectivity for Mycobacterial Proteasome Over Human Proteasomes: Role of S3 and S1 Binding Pockets. J. Am. Chem. Soc. 135, 9968-9971. doi:10.1021/ja400021x

Lobato-Márquez, D., Molina-García, L., Moreno-Córdoba, I., García-del Portillo, F., and Díaz-Orejas, R. (2016). Stabilization of the Virulence Plasmid pSLT of Salmonella Typhimurium by Three Maintenance Systems and Its Evaluation by Using a New Stability Test. Front. Mol. Biosci. 3, 66. doi:10.3389/fmolb.2016. 00066

Lunge, A., Gupta, R., Choudhary, E., and Agarwal, N. (2020). The Unfoldase ClpC1 of Mycobacterium Tuberculosis Regulates the Expression of a Distinct Subset of Proteins Having Intrinsically Disordered Termini. J. Biol. Chem. 295, 9455-9473. doi:10.1074/jbc.RA120.013456

Lupoli, T. J., Vaubourgeix, J., Burns-Huang, K., and Gold, B. (2018). Targeting the Proteostasis Network for Mycobacterial Drug Discovery. ACS Infect. Dis. 4, 478-498. doi:10.1021/acsinfecdis.7b00231

MacMicking, J. D., North, R. J., LaCourse, R., Mudgett, J. S., Shah, S. K., and Nathan, C. F. (1997). Identification of Nitric Oxide Synthase as a Protective Locus Against Tuberculosis. Proc. Natl. Acad. Sci. 94, 5243-5248. doi:10.1073/ pnas.94.10.5243

Marsee, J., Ridings, A., Yu, T., and Miller, J. (2018). Mycobacterium tuberculosis ClpC1 N-Terminal Domain Is Dispensable for Adaptor Protein-Dependent Allosteric Regulation. Int. J. Mol. Sci. 19, 3651. doi:10.3390/ijms19113651 
Masuda, H., Tan, Q., Awano, N., Wu, K.-P., and Inouye, M. (2012). YeeU Enhances the Bundling of Cytoskeletal Polymers of MreB and FtsZ, Antagonizing the CbtA (YeeV) Toxicity in Escherichia Coli. Mol. Microbiol. 84, 979-989. doi:10. 1111/j.1365-2958.2012.08068.x

Maurer, M., Linder, D., Franke, K. B., Jäger, J., Taylor, G., Gloge, F., et al. (2019). Toxic Activation of an AAA+ Protease by the Antibacterial Drug Cyclomarin A. Cel Chem. Biol. 26, 1169-1179. doi:10.1016/j.chembiol.2019.05.008

Melderen, L., Bernard, P., and Couturier, M. (1994). Lon-Dependent Proteolysis of CcdA Is the Key Control for Activation of CcdB in Plasmid-Free Segregant Bacteria. Mol. Microbiol. 11, 1151-1157. doi:10.1111/j.1365-2958.1994. tb00391.x

Molière, N., and Turgay, K. (2013). General and Regulatory Proteolysis in Bacillus Subtilis. Subcell Biochem. 66, 73-103. doi:10.1007/978-94-007-5940-4_4

Moreno-Cinos, C., Goossens, K., Salado, I. G., Van Der Veken, P., De Winter, H., and Augustyns, K. (2019). ClpP Protease, a Promising Antimicrobial Target. Int. J. Mol. Sci. 20, 2232. doi:10.3390/ijms20092232

Müller, A. U., Imkamp, F., and Weber-Ban, E. (2018). The Mycobacterial LexA/ RecA-Independent DNA Damage Response Is Controlled by PafBC and the Pup-Proteasome System. Cel Rep. 23, 3551-3564. doi:10.1016/j.celrep.2018. 05.073

Müller, A. U., and Weber-Ban, E. (2019). The Bacterial Proteasome at the Core of Diverse Degradation Pathways. Front. Mol. Biosci. 6, 23. doi:10.3389/fmolb. 2019.00023

Muthuramalingam, M., White, J., and Bourne, C. (2016). Toxin-Antitoxin Modules Are Pliable Switches Activated by Multiple Protease Pathways. Toxins 8, 214. doi:10.3390/toxins 8070214

Ogura, T., and Hiraga, S. (1983). Mini-F Plasmid Genes that Couple Host Cell Division to Plasmid Proliferation. Proc. Natl. Acad. Sci. 80, 4784-4788. doi:10. 1073/pnas.80.15.4784

Page, R., and Peti, W. (2016). Toxin-Antitoxin Systems in Bacterial Growth Arrest and Persistence. Nat. Chem. Biol. 12, 208-214. doi:10.1038/nchembio.2044

Pearce, M. J., Mintseris, J., Ferreyra, J., Gygi, S. P., and Darwin, K. H. (2008). Ubiquitin-Like Protein Involved in the Proteasome Pathway of Mycobacterium Tuberculosis. Science 322, 1104-1107. doi:10.1126/science.1163885

Pecota, D. C., and Wood, T. K. (1996). Exclusion of T4 Phage by the Hok/sok Killer Locus from Plasmid R1. J. Bacteriol. 178, 2044-2050. doi:10.1128/jb.178.7. 2044-2050.1996

Peltier, J., Hamiot, A., Garneau, J. R., Boudry, P., Maikova, A., Hajnsdorf, E., et al. (2020). Type I Toxin-Antitoxin Systems Contribute to the Maintenance of Mobile Genetic Elements in Clostridioides Difficile. Commun. Biol. 3, 718. doi:10.1038/s42003-020-01448-5

Prysak, M. H., Mozdzierz, C. J., Cook, A. M., Zhu, L., Zhang, Y., Inouye, M., et al. (2009). Bacterial Toxin YafQ Is an Endoribonuclease that Associates with the Ribosome and Blocks Translation Elongation Through Sequence-Specific and Frame-Dependent mRNA Cleavage. Mol. Microbiol. 71, 1071-1087. doi:10. 1111/j.1365-2958.2008.06572.x

Raju, R. M., Jedrychowski, M. P., Wei, J.-R., Pinkham, J. T., Park, A. S., O’Brien, K., et al. (2014). Post-translational Regulation via Clp Protease Is Critical for Survival of Mycobacterium Tuberculosis. PLoS Pathog. 10, e1003994. doi:10. 1371/journal.ppat.1003994

Ramage, H. R., Connolly, L. E., and Cox, J. S. (2009). Comprehensive Functional Analysis of Mycobacterium Tuberculosis Toxin-Antitoxin Systems: Implications for Pathogenesis, Stress Responses, and Evolution. PLoS Genet. 5, e1000767. doi:10.1371/journal.pgen.1000767

Ramisetty, B. C. M. (2020). Regulation of Type II Toxin-Antitoxin Systems: The Translation-Responsive Model. Front. Microbiol. 11, 895. doi:10.3389/fmicb. 2020.00895

Ruangprasert, A., Maehigashi, T., Miles, S. J., and Dunham, C. M. (2017). Importance of the E. coli DinJ Antitoxin Carboxy Terminus for Toxin Suppression and Regulated Proteolysis. Mol. Microbiol. 104, 65. doi:10.1111/ mmi.13641

Sala, A., Bordes, P., and Genevaux, P. (2014). Multiple Toxin-Antitoxin Systems in Mycobacterium Tuberculosis. Toxins 6, 1002-1020. doi:10.3390/toxins6031002

Sala, A. J., Bordes, P., Ayala, S., Slama, N., Tranier, S., Coddeville, M., et al. (2017). Directed Evolution of SecB Chaperones Toward Toxin-Antitoxin Systems. Proc. Natl. Acad. Sci. U.S.A. 114, 12584-12589. doi:10.1073/pnas.1710456114

Samanovic, M. I., Tu, S., Novák, O., Iyer, L. M., McAllister, F. E., Aravind, L., et al. (2015). Proteasomal Control of Cytokinin Synthesis Protects Mycobacterium
Tuberculosis Against Nitric Oxide. Mol. Cel 57, 984-994. doi:10.1016/j.molcel. 2015.01.024

Sassetti, C. M., Boyd, D. H., and Rubin, E. J. (2003). Genes Required for Mycobacterial Growth Defined by High Density Mutagenesis. Mol. Microbiol. 48, 77-84. doi:10.1046/j.1365-2958.2003.03425.x

Sauer, R. T., and Baker, T. A. (2011). AAA+ Proteases: ATP-Fueled Machines of Protein Destruction. Annu. Rev. Biochem. 80, 587-612. doi:10.1146/annurevbiochem-060408-172623

Schuessler, D. L., Cortes, T., Fivian-Hughes, A. S., Lougheed, K. E. A., Harvey, E., Buxton, R. S., et al. (2013). Induced Ectopic Expression of HigB Toxin in M Ycobacterium Tuberculosis Results in Growth Inhibition, Reduced Abundance of a Subset of mRNAs and Cleavage of tmRNA. Mol. Microbiol. 90, 195-207. doi: $10.1111 / \mathrm{mmi} .12358$

Sherrid, A. M., Rustad, T. R., Cangelosi, G. A., and Sherman, D. R. (2010). Characterization of a Clp Protease Gene Regulator and the Reaeration Response in Mycobacterium Tuberculosis. PLoS One 5, e11622. doi:10.1371/ journal.pone.0011622

Songailiene, I., Juozapaitis, J., Tamulaitiene, G., Ruksenaite, A., Šulčius, S., Sasnauskas, G., et al. (2020). HEPN-MNT Toxin-Antitoxin System: The HEPN Ribonuclease Is Neutralized by OligoAMPylation. Mol. Cel 80, 955-970. doi:10.1016/j.molcel.2020.11.034

Srinivasan, R., Anilkumar, G., Rajeswari, H., and Ajitkumar, P. (2006). Functional Characterization of AAA Family FtsH Protease of Mycobacterium Tuberculosis. FEMS Microbiol. Lett. 259, 97-105. doi:10.1111/j.1574-6968. 2006.00251.x

Tandon, H., Sharma, A., Wadhwa, S., Varadarajan, R., Singh, R., Srinivasan, N., et al. (2019). Bioinformatic and Mutational Studies of Related ToxinAntitoxin Pairs in Mycobacterium Tuberculosis Predict and Identify Key Functional Residues. J. Biol. Chem. 294, 9048-9063. doi:10.1074/jbc. RA118.006814

Texier, P., Bordes, P., Nagpal, J., Sala, A. J., Mansour, M., Cirinesi, A.-M., et al. (2021). ClpXP-Mediated Degradation of the TAC Antitoxin Is Neutralized by the SecB-like Chaperone in Mycobacterium tuberculosis. J. Mol. Biol. 433, 166815. doi:10.1016/j.jmb.2021.166815

Tiwari, P., Arora, G., Singh, M., Kidwai, S., Narayan, O. P., and Singh, R. (2015). MazF Ribonucleases Promote Mycobacterium Tuberculosis Drug Tolerance and Virulence in guinea Pigs. Nat. Commun. 6, 6059. doi:10.1038/ncomms7059

Trentini, D. B., Suskiewicz, M. J., Heuck, A., Kurzbauer, R., Deszcz, L., Mechtler, K., et al. (2016). Arginine Phosphorylation Marks Proteins for Degradation by a Clp Protease. Nature 539, 48-53. doi:10.1038/nature20122

Vahidi, S., Ripstein, Z. A., Juravsky, J. B., Rennella, E., Goldberg, A. L., Mittermaier, A. K., et al. (2020). An Allosteric Switch regulates Mycobacterium Tuberculosis ClpP1P2 Protease Function as Established by Cryo-EM and Methyl-TROSY NMR. Proc. Natl. Acad. Sci. USA 117, 5895-5906. doi:10.1073/pnas. 1921630117

Van Melderen, L. (2010). Toxin-antitoxin Systems: Why So Many, What for?. Curr. Opin. Microbiol. 13, 781-785. doi:10.1016/j.mib.2010.10.006

Wang, J., McIntosh, F., Radomski, N., Dewar, K., Simeone, R., Enninga, J., et al. (2015). Insights on the Emergence of Mycobacterium Tuberculosis from the Analysis of Mycobacterium Kansasii. Genome Biol. Evol. 7, 856-870. doi:10. 1093/gbe/evv035

Wang, X., Lord, D. M., Cheng, H.-Y., Osbourne, D. O., Hong, S. H., SanchezTorres, V., et al. (2012). A New Type V Toxin-Antitoxin System where mRNA for Toxin GhoT Is Cleaved by Antitoxin GhoS. Nat. Chem. Biol. 8, 855-861. doi:10.1038/nchembio.1062

Wilmaerts, D., Bayoumi, M., Dewachter, L., Knapen, W., Mika, J. T., Hofkens, J., et al. (2018). The Persistence-Inducing Toxin HokB Forms Dynamic Pores That Cause ATP Leakage. mBio 9, e00744. doi:10.1128/mBio.00744-18

Wilmaerts, D., Dewachter, L., De Loose, P.-J., Bollen, C., Verstraeten, N., and Michiels, J. (2019). HokB Monomerization and Membrane Repolarization Control Persister Awakening. Mol. Cel 75, 1031-1042. doi:10.1016/j.molcel. 2019.06.015

Xie, Y., Wei, Y., Shen, Y., Li, X., Zhou, H., Tai, C., et al. (2018). TADB 2.0: An Updated Database of Bacterial Type II Toxin-Antitoxin Loci. Nucleic Acids Res. 46, D749-D753. doi:10.1093/nar/gkx1033

Yamada, Y., and Dick, T. (2017). Mycobacterial Caseinolytic Protease Gene Regulator ClgR Is a Substrate of Caseinolytic Protease. mSphere 2, e00338. doi:10.1128/mSphere.00338-16 
Yamaguchi, Y., Park, J.-H., and Inouye, M. (2011). Toxin-antitoxin Systems in Bacteria and Archaea. Annu. Rev. Genet. 45, 61-79. doi:10.1146/annurev-genet110410-132412

Ye, F., Li, J., and Yang, C.-G. (2016). The Development of Small-Molecule Modulators for ClpP Protease Activity. Mol. BioSyst. 13, 23-31. doi:10.1039/ c6mb00644b

Yu, X., Gao, X., Zhu, K., Yin, H., Mao, X., Wojdyla, J. A., et al. (2020). Characterization of a Toxin-Antitoxin System in Mycobacterium Tuberculosis Suggests Neutralization by Phosphorylation as the Antitoxicity Mechanism. Commun. Biol. 3, 216. doi:10.1038/s42003-020-0941-1

Zaveri, A., Wang, R., Botella, L., Sharma, R., Zhu, L., Wallach, J. B., et al. (2020). Depletion of the DarG Antitoxin in Mycobacterium Tuberculosis Triggers the DNA-damage Response and Leads to Cell Death. Mol. Microbiol. 114, 641-652. doi:10.1111/mmi.14571

Zhan, W., Hsu, H.-C., Morgan, T., Ouellette, T., Burns-Huang, K., Hara, R., et al. (2019). Selective Phenylimidazole-Based Inhibitors of the Mycobacterium Tuberculosis Proteasome. J. Med. Chem. 62, 9246-9253. doi:10.1021/acs. jmedchem.9b01187

Zhang, S., Burns-Huang, K. E., Janssen, G. V., Li, H., Ovaa, H., Hedstrom, L., et al. (2017). Mycobacterium tuberculosis Proteasome Accessory Factor A (PafA) Can Transfer Prokaryotic Ubiquitin-Like Protein (Pup) Between Substrates. mBio 8, e00122. doi:10.1128/mBio.00122-17
Zhou, X., Eckart, M. R., and Shapiro, L. (2021). A Bacterial Toxin Perturbs Intracellular Amino Acid Balance To Induce Persistence. mBio 12, e03020. doi:10.1128/mBio.03020-20

Ziemski, M., Jomaa, A., Mayer, D., Rutz, S., Giese, C., Veprintsev, D., et al. (2018). Cdc48-like Protein of Actinobacteria (Cpa) Is a Novel Proteasome Interactor in Mycobacteria and Related Organisms. elife 7, e34055. doi:10.7554/eLife.34055

Ziemski, M., Leodolter, J., Taylor, G., Kerschenmeyer, A., and Weber-Ban, E. (2020). Genome-wide Interaction Screen for Mycobacterium Tuberculosis ClpCP Protease Reveals Toxin-Antitoxin Systems as a Major Substrate Class. FEBS J. 288, 99-114. doi:10.1111/febs.15335

Conflict of Interest: The authors declare that the research was conducted in the absence of any commercial or financial relationships that could be construed as a potential conflict of interest.

Copyright (C) 2021 Bordes and Genevaux. This is an open-access article distributed under the terms of the Creative Commons Attribution License (CC BY). The use, distribution or reproduction in other forums is permitted, provided the original author(s) and the copyright owner(s) are credited and that the original publication in this journal is cited, in accordance with accepted academic practice. No use, distribution or reproduction is permitted which does not comply with these terms. 